\title{
Tuna may have efficient hydrodynamic multiple small fins for stability
}

\author{
yoshiyasu takefuji ${ }^{1}$
}

${ }^{1}$ Affiliation not available

September 21, 2020

Dylan K Wainwright et al. indicated high-performance Tuna platform for designing autonomous underwater vehicles [1]. Although their research will be useful, they should focus on the role of fins. Vadim Pavlov, et al. described hydraulic control of tuna fins [2]. During the late $18^{\text {th }}$ century, wing designs were based on anatomical studies on dolphins, trout and tuna by the "father of aerodynamics", Sir George Cayley [3,4]. Fish that are adapted for fast swimming, like tuna, are generally fusiform in shape and this is considered an efficient, hydrodynamic shape [5,6]. The hydraulic-fin-manufacture utilized multiple small fins instead of large hydraulic fins through the experiment for adopting better boat stabilizers [7]. By using two, four or six smaller active fins grouped together, the more small fins system produces better results without causing drag and slowing boats [7]. Tuna has such efficient hydrodynamic multiple small fins including 1st dorsal fin, 2nd dorsal fin, finlets, caudal fin, pelvic fin, pectoral fin, and anal fin. Every tuna fin may have the special role for better performance in stabilization of any vehicles.

\section{References:}

1. Dylan K Wainwright and George V Lauder 2020 Bioinspir. Biomim.15 035007

2. Vadim Pavlov, et al., Hydraulic control of tuna fins: A role for the lymphatic system in vertebrate locomotion, Science, 21 Jul 2017, Vol.357, Issue 6348, pp.310-314

3. Bio-mimetic Drag Reduction - Part 2: Aero- and Hydrodynamics, March 31, 2012

http://aerospaceengineeringblog.com/bio-mimetic-drag-reduction-2/

4. https://en.wikipedia.org/wiki/George_Cayley

5. Eric Pickhartz, The 'Tuna Robot' is the Navy's Newest Underwater Drone, July 4, 2014

http://www.wideopenspaces.com/tuna-robot-navys-newest-underwater-drone/

6. Kirk Janowiak, Are fish aerodynamic?, Jan 12, 2015

https://www.quora.com/Are-fish-aerodynamic

7. High-Performance Active Fin Stabilizers Improve Boat Speed and Performance, Feb 21, 2017

http://www.gyrogalestabilizers.com/high-performance-active-fin-stabilizers-improve-boat-speedperformance/ 